\title{
An evaluation of oxygen therapy equipment Experimental study of various devices on the human subject
}

\author{
D. W. BETHUNE AND J.M.COLLIS
}

From the Department of Anaesthesia, St. Bartholomew's Hospital, London, E.C.1

\begin{abstract}
Six commercially available devices were tested to determine the alveolar oxygen concentration that is achieved and the volume of rebreathing that can occur in conditions simulating clinical use. All were tested at zero oxygen flow, since failure and interruption of the oxygen supply to such devices can occur in clinical use. In patients whose cardiorespiratory reserve is exhausted, any increase in dead space will be detrimental ; for such patients devices should be selected which cannot cause rebreathing under any circumstances. Three of the devices tested could cause appreciable rebreathing, i.e., Oxyaire (B.L.B. pattern), Pneumask, and the M.C. mask. Claims made for the Pneumask and the M.C. mask were not substantiated in this study. With administration by nasal catheter changes in alveolar oxygen concentrations were found to be dependent on the percentage of mouth breathing by the subject. The Ventimask produced a consistent elevation of the alveolar oxygen percentage and did not cause any increase in the dead space.
\end{abstract}

The administration of oxygen is a recognized method of treatment in a variety of clinical conditions. The authors formed the impression that an undesirable amount of rebreathing with some types of oxygen mask was possible in practice. The present study was undertaken to permit quantitative investigations of the volume of rebreathing as well as of the alveolar oxygen percentage obtained with a variety of devices.

\section{PREVIOUS STUDIES}

There has been a wide variation in the methods used for testing oxygen therapy equipment ; Kory, Bergmann, Sweet, and Smith (1962) gave an extensive bibliography and concluded that alveolar sampling is the most reliable index of performance.

In their results they gave values for alveolar $\mathrm{PCO}_{2}$ but commented that ventilation would be adjusted to overcome the effects of rebreathing in studies similar to theirs using normal subjects; ventilation was not measured. An earlier paper by Cotes (1956) estimated the increase in ventilation using oxygen masks fitted with reservoir bags. A valve on the reservoir could be used to prevent rebreathing. As far as we know, this is the only study which has attempted to measure the increase in effective dead space. Other authors have expressed widely varying opinions on this topic (Catterall and Snow, 1960 ; Catterall, 1960 ; and Campbell, 1960). The method described by Cotes (1956) is not applicable to devices without rebreathing bags.

Other papers have used oxygen saturation estimated by oximetry (Ball, 1963) and mechanical models (Flenley, Hutchison, and Donald, 1963) to estimate the efficiency of equipment in delivering oxygen.

\section{METHOD}

A stethograph, made from a modified pneumatic body splint $^{1}$ (Fig. 1), was arranged to drive a potentiometric recorder visible to the subject; the system was calibrated against a Parkinson Cowan Dry Gas Meter (C.D.I.). The subject maintained a constant tidal volume by observing the potentiometric recorder, previously calibrated at 500 and $1,000 \mathrm{ml}$. The rate of respiration (f) was obtained from the tracing. End tidal samples, obtained by a modification of the Haldane Priestley method, were analysed for oxygen and carbon dioxide tensions by Clarke and Severinghaus electrodes. ${ }^{2}$ The subjects in this study were free from cardiorespiratory disease.

Using the values obtained for ventilation and end tidal $\mathrm{PCO}_{2}$, the physiological dead space for any

1 Oxygenaire Ltd.

${ }^{2}$ Radiometer Ltd., Copenhagen 


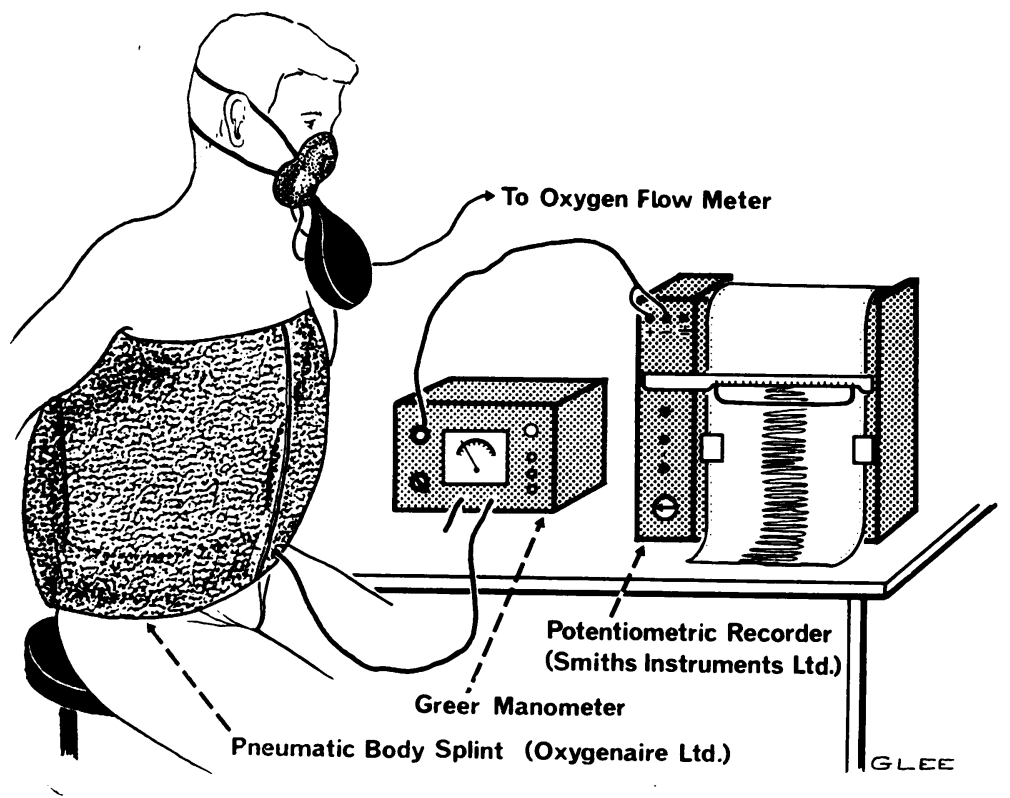

FIG. 1 .

subject-equipment combination can be obtained from :

$$
\mathrm{VD}(\text { total })=\frac{\dot{\mathrm{VE}}-\left(\frac{\mathrm{CO}_{2} \text { evolved } / \mathrm{min} .}{\mathrm{FACO}}\right)}{\mathrm{f}}
$$

where $\mathrm{VD}=$ dead space volume (ml.); $\dot{\mathrm{VE}}=$ minute ventilation (ml.) ; FA $\mathrm{CO}_{2}=$ fraction $\mathrm{CO}_{2}$ in alveolar gas ; $f=$ respiratory rate per minute.

The subject's dead space and $\mathrm{CO}_{2}$ production per minute were calculated from independent measurements. Subtraction of the subject's dead space from the total dead space when using a mask will give the effective dead space of the mask under test. Determinations were made at zero flow and with oxygen flows of $1,2,4$, and 8 litres per minute, time being allowed before each estimation to allow equilibration to occur.

\section{RESULTS}

Six devices were tested by this method (Fig. 2).

The increase in ventilation with the Pneumask compensates for the increase in effective dead space, to maintain an end tidal $\mathrm{PCO}_{2}$ within normal range (Fig. 3). Similar changes in end tidal $\mathrm{PCO}_{2}$ were observed by Kory et al. (1962) also using normal subjects. These results with the Ventimask and Pneumask serve to illustrate the importance of the simultaneous measurement of ventilation and $\mathrm{PCO}_{2}$. The subjects tended to hyperventilate at high oxygen flows, but this does not affect the calculation for dead space whew FA $\mathrm{CO}_{2}, \dot{\mathrm{VE}}$, and $\mathrm{f}$ are all known.

EFFECTIVE DEAD SPACE Figure 4 shows the effec $\overrightarrow{\overrightarrow{\hat{\rho}}}$ tive dead space obtained with the six devices.

Pneumask This has a larger dead space at alp. oxygen flows than the other devices tested. The soft plastic moves in and out with each respiration giving a 'flip flop' effect. This is a reassuring sign of respiration, but the volume of rebreathing is high.

Subjective discomfort occurred at low oxygers flows probably related to the hyperventilation at a fixed tidal volume of $500 \mathrm{ml}$.

Oxyaire mask The rebreathing bag fitted to the. mask we tested collapsed of its own accord at the end of expiration, and this probably produced lower figures for the effective dead space than would a softer rebreathing bag.

M.C. mask It has been claimed (Catterall, 1960) that the M.C. mask has no dead space, due to के 'continuous and organized movement of the gases? inside the mask'. This claim was not substantiatedo in our study. The effective dead space at the recommended flow of $21 . / \mathrm{min}$. was calculated to be $75 \mathrm{ml}$.

Ventimask This has a minimal effective deack space; the fall to a negative effective dead spaces 


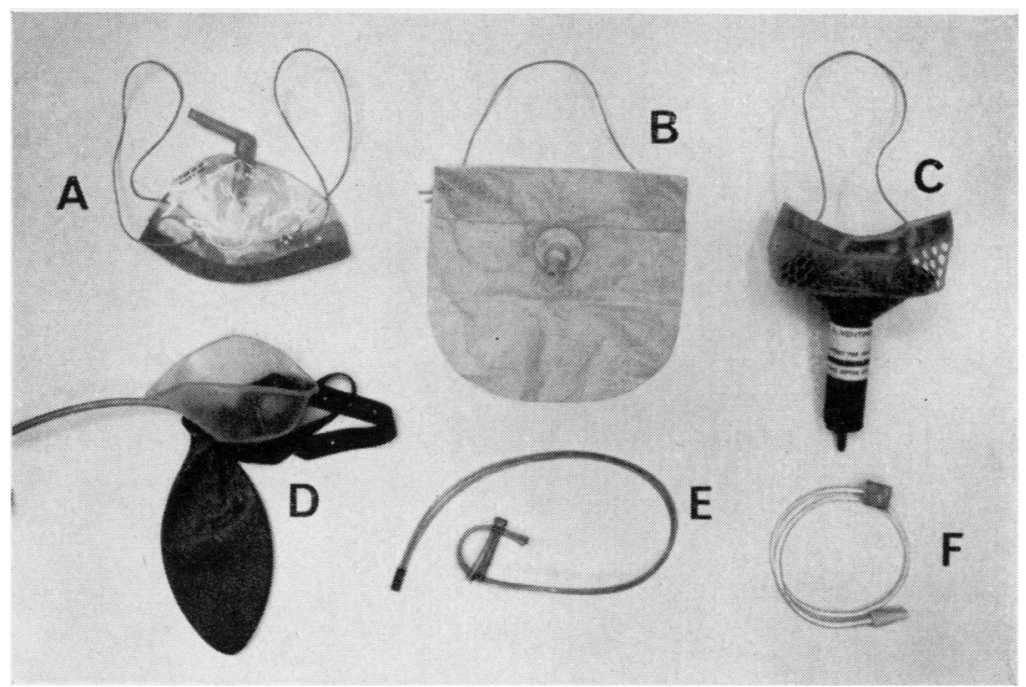

FIG. 2. Anaesthetic masks and apparatus: (A) M.C. mask, Bakelite Xyonite Ltd.; (B) Pneumask (medium), Oxygenaire Ltd.; (C) Ventimask (24\%), Oxygenaire Ltd.; (D) Oxyaire (large), Oxygenaire Ltd.; $(E)$ double nasal catheters, Eschmann Bros Ltd.; $(F)$ single nasal catheter with compress (Norwegian).

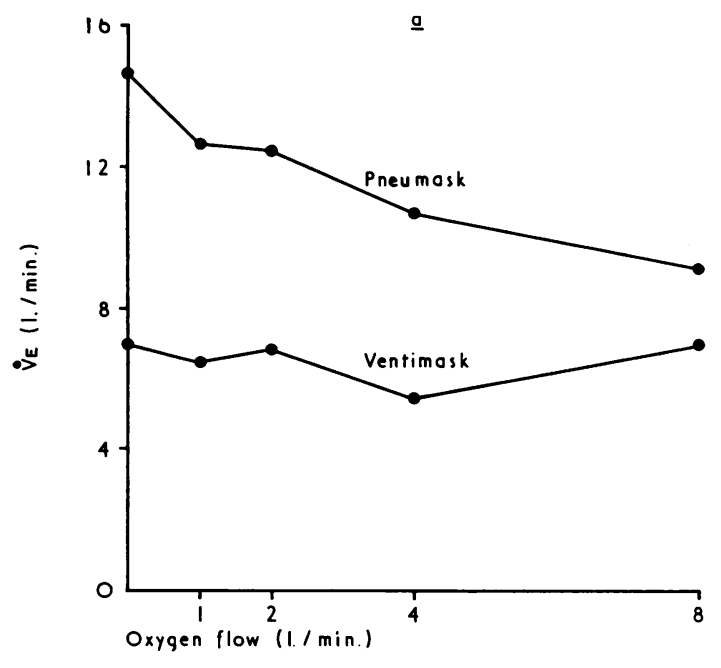

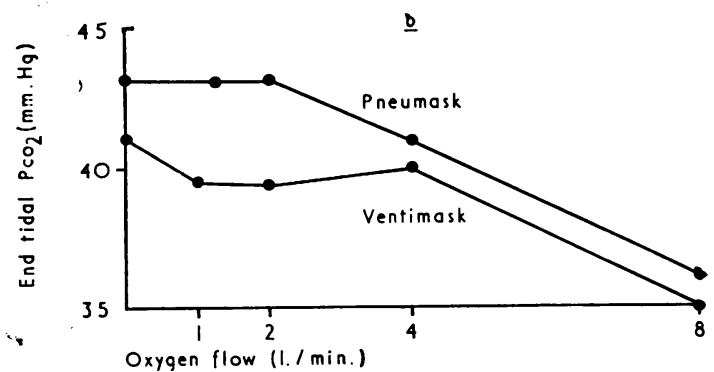

FIG. 3. Graphs to show results obtained from which effective dead space can be calculated by applying equation (1).

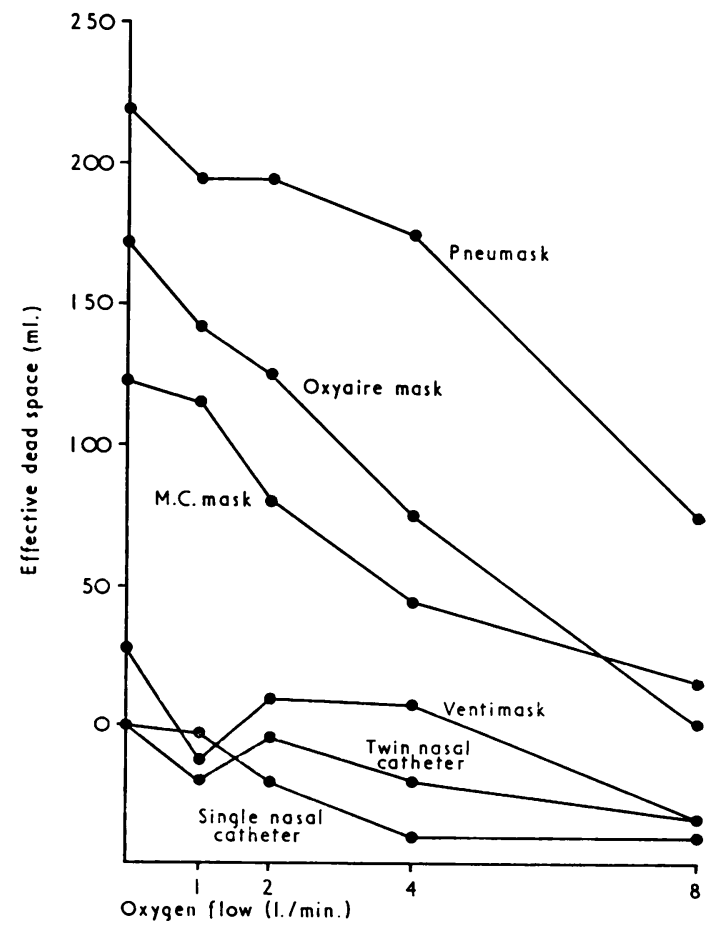

FIG. 4. Effective dead space in millilitres. Determinations made with $500 \mathrm{ml}$. tidal volume. 
at high $\mathrm{O}_{2}$ flows is presumably due to a high flow of air/oxygen mixture past the face. At 8 $1 . / \mathrm{min}$. $\mathrm{O}_{2}$ this is over $200 \mathrm{l}$. of gas mixture per minute.

Nasal catheters These cause a reduction in the effective dead space, presumably due to flushing of the nasal passage with oxygen at all flow rates.

ALVEOLAR OXYGEN PERCENTAge (Figs 5 to 7) These results for alveolar oxygen percentage agree in general with those in the literature. We found major differences, as illustrated (Fig. 5), between mouth and nose breathing with nasal catheters.

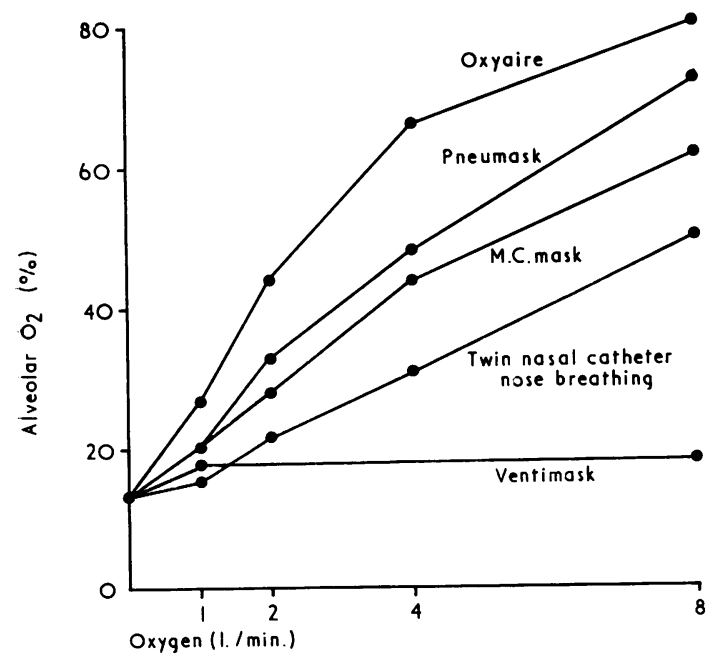

FIG. 5. Percentage alveolar oxygen, tidal volume $500 \mathrm{ml}$.

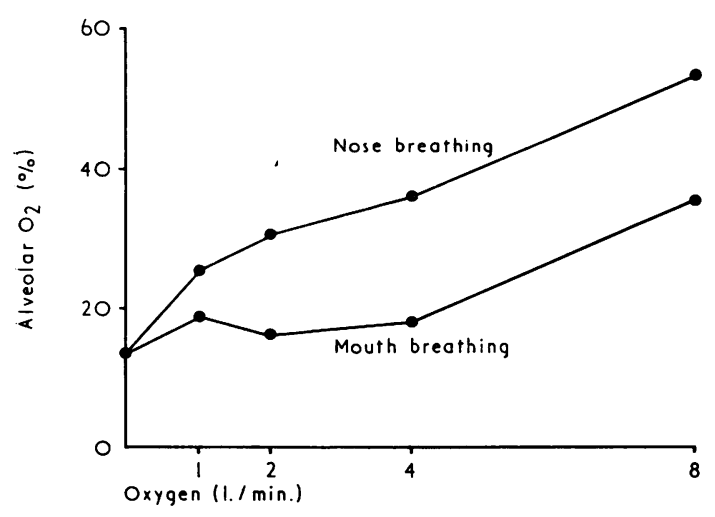

FIG. 6. Effect of mode of breathing on alveolar oxygen percentage, single nasal catheter, tidal volume $500 \mathrm{ml}$.

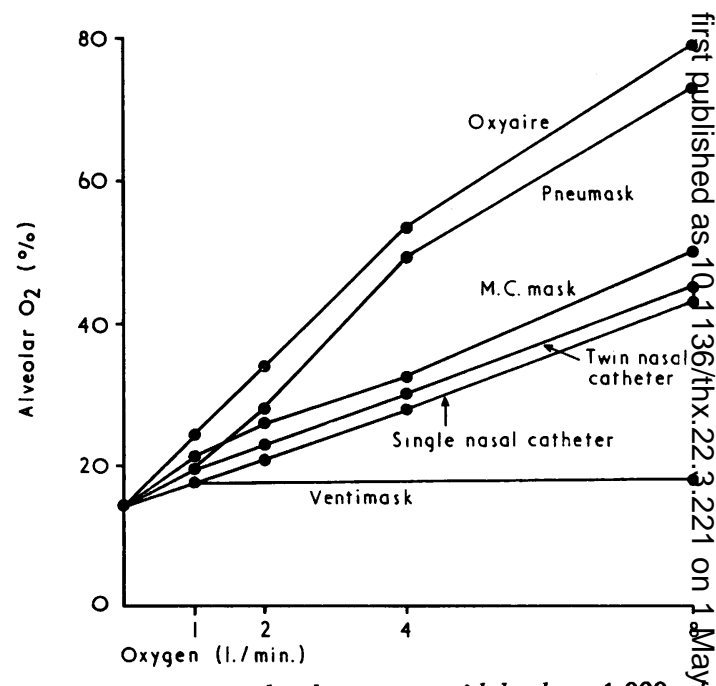

FIG. 7. Percentage alveolar oxygen, tidal volume $1,000 \mathrm{mI}$.

The Venturi principle of the Ventimask gives 2 very consistent alveolar oxygen concentration The B.L.B. pattern mask (Oxyaire) is capable of giving high oxygen levels if it is a good fit, as was in this case.

\section{DISCUSSION}

The methods used in this study allow estimatio of the effective dead space of oxygen therapy equipment with the subject breathing at a con stant tidal volume. The method, using a stethos graph and end tidal sampling, gave consisteny results, but practice was necessary to keep the tidal volume constant in the face of the large changes in minute ventilation which occurreg with some masks. End tidal sampling has beef criticized for estimating alveolar concentrations when a mask has to be removed, as there is $\frac{\mathrm{d}}{\mathrm{S}}$ period of breath-holding during this manœuvre (Flenley et al., 1963). We feel that the shof period, less than 2 seconds, before the sampling is started does not invalidate our results. Wes attempted spot sampling and found an unsatisfač tory scatter in the values obtained; this scatter has been observed by previous authors (Kory al., 1962; Flenley et al., 1963). The results wo obtained by end tidal sampling were consistent on repetition.

Three of the devices tested have had clain made for their function.

Ventimask This gives a consistent alveolar cop centration over a wide range of oxygen flow rate8 
Positional changes did not produce significant changes. Our results agree with those of Campbell and Gebbie (1966). Unfortunately the mask is manufactured in opaque plastic and the patient's face is partially obscured. In our opinion, this type of mask is the only one that we tested which is suitable for controlled oxygen therapy. Separate Ventimasks to deliver $24 \%, 28 \%$, and $35 \%$ inspired oxygen are available.

Safety Pneumask This is probably the type of device for oxygen therapy most commonly used in the United Kingdom. The claim that if the oxygen supply fails the cushion deflates and the mask falls off was not substantiated. An elastic band is provided which holds the mask securely in place. In our opinion, the presence of a large dead space if the oxygen supply fails makes this device potentially dangerous.

M.C. mask The claim that the design of the mask prevents the formation of any dead space was not confirmed in this study. With zero flow the increase in effective dead space is over $100 \mathrm{ml}$. At the recommended flow of $21 . / \mathrm{min}$. it would still be significant in a patient with impaired cardiopulmonary reserve.

We could discover no specific claims for the other three devices tested. The Oxyaire mask is capable of giving high alveolar oxygen levels and would be useful when a high oxygen level is required. A high fresh gas flow, of at least 8 $1 . /$ min., will be needed to achieve this. In view of the large increase in effective dead space if the oxygen fails it should only be used under supervision. At lower oxygen flows we agree with the remark made by Cotes (1956) that this type of mask 'should be reserved for conditions in which some inhalation of $\mathrm{CO}_{2}$ is also desirable'. The two methods of giving nasal oxygen tested did not retain $\mathrm{CO}_{2}$ and there could be no increase in the effective dead space. There was a marked difference in the alveolar oxygen, depending on the degree of mouth and nose breathing.

\section{CONCLUSION}

The results obtained in this study confirmed the impression that some pieces of equipment used to administer oxygen therapy could cause rebreathing if the oxygen flow failed or was reduced. Hyperventilation will compensate for the rebreathing in a normal subject even with the worst device tested. However, patients who require oxygen therapy may not have a cardiopulmonary reserve to compensate for any increase in effective dead space. Post-operative (anaesthetic) hypoxaemia can be corrected by an inspired mixture of $30 \%$ oxygen (Nunn and Payne, 1962). This can be achieved by five of the devices tested at an oxygen flow of 2 litres/minute. (The Ventimask tested was designed to give $24 \%$ inspired oxygen.) The nasal catheters could not be relied on, as patients may mouth breathe. It is unfortunate that all the other devices tested had appreciable dead spaces at flows of 2 litres/ minute.

We should like to thank Dr. Peter Cole for the use of the laboratory and for technical advice, and Mr. L. Hawkins for his assistance with the gas analysis; the Joint Research Board for financial assistance ; Dr. T. B. Boulton for help and encouragement in the presentation of this paper; and Miss $J$. Goodwin and Miss E. O'Sullivan for secretarial assistance.

\section{ADDENDUM}

A recent article by Catterall, Kazantzis, and Hodges (1967) admits the presence of a dead space in the M.C. mask and estimates it to be approximately $90 \mathrm{ml}$.

\section{REFERENCES}

Ball, J. A. C. (1963). Oxygen masks. Brit. J. Anaesth., 35, 368.

Campbell, E. J. M. (1960). A method of controlled oxygen administration which reduces the risk of carbon-dioxide retention. Lancet, 2 , 12.

and Gebbie, T. (1966). Masks and tents for providing controlled oxygen concentrations. Ibid., 1, 468

Catterall, M. (1960). A new mask for delivering oxygen or other gases. Brit. med. J., 1, 1941.

and Snow, M. (1960). The Polymask as a means of administering oxygen. Ibid., 1, 1254.

Kazantzis, G., and Hodges, B. (1967). Performance of nasal catheters and a face mask in oxygen therapy. Lancet, 1, 415.

Cotes, J. E. (1956). Reassessment of value of oxygen masks that permit rebreathing. Brit. med. J., 1, 269.

Flenley, D. C., Hutchison, D. C. S., and Donald, K. W. (1963) Behaviour of apparatus for oxygen administration. Ibid., 2, 1081.

Kory, R. C., Bergmann, J. C., Sweet, R. D., and Smith, J. R. (1962). Comparative evaluation of oxygen therapy techniques. $J$. Amer. med. Ass., 179, 767.

Nunn, J. F., and Payne, J. P. (1962). Hypoxaemia after general anaesthesia. Lancet, 2,631 . 